EPJ manuscript No.

(will be inserted by the editor)

\title{
Gradual learning and the evolution of cooperation in the spatial Continuous Prisoner's Dilemma
}

\author{
Raúl Jiménez ${ }^{1 a}$, Haydée Lugo ${ }^{2}$, and Maxi San Miguel $^{3}$ \\ 1 Departamento de Estadística, Universidad Carlos III de Madrid, 28903 Madrid, Spain \\ 2 Departamento de Economía, Universidad Carlos III de Madrid, 28903 Madrid, Spain \\ 3 IFISC, Instituto de Física Interdisciplinar y Sistemas Complejos (CSIC-UIB), E-07122 Palma de Mallorca, Spain
}

Received: date / Revised version: date

\begin{abstract}
The usual mechanism for modeling learning in spatially structured evolutionary games has to date been imitation of some successful neighbor. However, it seems natural that individuals hesitate to imitate their neighbor's acts, specially if they can imply high costs. Here we study the effect of incorporating resistance to imitation on these models. Our framework is the spatial Continuous Prisoner's Dilemma. For this evolutionary game, it has been reported that occasional errors in the imitation process can explain the emergence of cooperation from a non-cooperative initial state. In this work, we show that this only occurs for particular regimes of low costs of cooperation. Furthermore, we display how resistance gets greater the range of scenarios where cooperative individuals can invade selfish populations. In this context, where resistance to imitation can be interpreted as a general rule of gradual learning, our results show that the less that is learnt in a single step from a successful neighbors, the larger the degree of global cooperation finally attained. In general, the effect of step-by-step learning can be more efficient for the evolution of cooperation than a full blast one.
\end{abstract}

PACS. PACS-02.50Le Decision theory and game theory - PACS-89.75Fb Structures and organization in complex systems

\section{Introduction}

Send offprint requests to: Raúl Jiménez

The evolution of cooperation has been a challenging prob-

a e-mail: raul.jimenez@uc3m.es

lem since Darwin [1-5]. With sophisticated tools of game 
theory, researchers from many fields have attempted to shed light on the underlying mechanisms that outperform the vulnerability of cooperation of being cheated [6]. Models with spatial structures have been considered by these researches to mimic real population where individuals do not interact with everybody else. In these models, individuals are located on the nodes of a network, play repeatedly with their neighbors, and update their strategies by imitating (with occasional errors) the strategy of some more successful neighbor. Different update rules respond to the same evolutionary principle of reproduction of successful strategies [7] and are usually implemented through two basic operators: Selection, in which individuals identify whom to imitate, and mutation, occasional errors in the imitation process.

Discrete-choice models, where cooperation is all or nothing, do not lead mutation to generating new strategies into the population. However in models in which individuals can exhibit variable degrees of cooperation, mutation can be certainly a source of generating diversity. The study of cooperative behavior in a quantitative way rather than a qualitative way has been crucial in dealing with the problem of evolution and the stability of cooperation. The first studies on this issue $[8,9]$ considered the evolution of degrees of cooperation by interpolating payoffs between the discrete outcomes of the classical Prisoner's Dilemma model (PD). A natural approach to model variable levels of cooperation was introduced later $[10,11]$. Iteration and spatial structures based on continuous cooperative investment have also been addressed [12-19], [20,21] provide a complete review of previous researches on the stability in cooperation in variable-investment systems. Developing some of the ideas of [10], and following the classical scheme of [22] for spatial evolutionary games, Killingback et al. [12] introduced the spatial Continuous Prisioner's Dilemma (CPD). The joint consideration of continuous cooperative investments and spatial structured populations in their model represents an important advance in explaining the evolutionary origin of cooperation [21].

The strategy update rule considered in [22] was originally designed for the discrete PD, where exact imitation is the only way to update strategies. In a social and economic context, where imitation is interpreted as learning [19], it seems natural that individuals hesitate to imitate exactly the investment of a neighbor. In this work we investigate the effect of introducing some grade of resistance to imitation in the spatial CPD. This resistance leads to a process in which individuals learn step by step from their successful neighbors. For this purpose we employ the most elemental rule that can be used: Each individual updates her investment by averaging her current investment with the investment of her successful neighbor. In adverse conditions in which costs for cooperation are high, individuals have reason to avoid the blind imitation of higher-investors, despite how successful they can be. Counter-intuitively, we show that this wariness leads partial learning to being more efficient than a full blast imitation precisely in regimes of non-low costs of cooperation. The paper is organized as follows: In section 2 , we review the spatial CPD as well as we introduce gen- 
eral assumptions and the notation used throughout the paper. In section 3, we characterize the different payoff regimes according to the asymptotic behavior of the spatial CPD. In particular, we prove that previous results $[12, ?]$ in which it is reported that cooperation gradually increases from a non-cooperative state correspond to particular payoff regimes that we classify as regimes of low cost. In section 4 , we introduce resistance to imitation and explain how this mechanism operates for the maintenance and promotion of cooperation. We also provide the conditions in which higher-investment individuals can invade a population of selfish individuals. Section 5 summaries our conclusions.

\section{The CPD in spatially structured}

\section{populations}

In the CPD, if an individual, who invests $x$, interacts with another, who invests $y$, the former receives a payoff equal to $S(x, y)=B(y)-C(x)$ and the latter $S(y, x)=$ $B(x)-C(y)$. The function $B(\cdot)$ specifies the benefit that an individual obtains from the investment made by the other in their pairwise interaction, and the function $C(\cdot)$ specifies the cost incurred by her. As standard assumptions, functions $B(\cdot)$ and $C(\cdot)$ are increasing and concave, with $B(0)=C(0)=0$, and $B(x) \geq C(x)$ for any feasible investment $x[21]$. In order to determine the maximal possible investment, it is required to compute the optimal mutual investment $x_{\max }$ that maximizes $B(x)-C(x)$. In general, for all $0<x<y<x_{\max }$, one can verify the inequalities shown by [12]

$$
S(y, x)<S(x, x)<S(y, y)<S(x, y)
$$

which are continuous versions of the well known conditions for the payoffs of the classical Prisoner's Dilemma [17].

To avoid the problem of comparing investments in different scales, we only consider investments in $[0,1]$. For that, it is sufficient to have $x_{\max } \geq 1$, therefore (1) holds for any pair of investments $0<x<y<1$.

Although the main results of this work cover general increasing-concave functions, the simulations shown throughout this paper are based on linear costs, namely, $C(x)=$ $C x$, and benefit functions of the form $B(x)=a[1-\exp (-b x)]$, with $a, b>0$. These functions are typical of what might be expected in real biological situations $[23,24]$ and are basis functions in the literature of the CPD $[12,17,19]$. For these basis functions, one has $x_{\max }=-\log (C /(a b)) / b$, thus, $x_{\max } \geq 1$ if and only if $C<a b \exp (-b)$. In our parametric analysis, we fix $a$ and $b$ and vary $C$ between 0 and $a b \exp (-b)$. One additional remark:

$$
a\left(1-\exp ^{-b x}\right) \rightarrow B x
$$

when $b \rightarrow 0^{+}$and $a \rightarrow+\infty$, with $a b \rightarrow B<\infty$. In fact, for any investment $0<x<1$, the convergence is very fast. Therefore, linear benefit functions, used by [13,14], are an interesting limit case of the basis functions and, because of their simplicity, are especially considered in our analysis.

In the spatial CPD, individuals are placed on the nodes of a network and recollect payoffs from their pairwise interactions with their neighbors accordingly with a CPD. The total payoff of each individual is given by the sum of 
these payoffs. At each time step, each individual updates her investment by imitating the investment of her neighbor (including herself) with the highest total payoff. Additionally, and this is an important ingredient in the model, [12] consider occasional errors at the imitation moment (mutations) that can change the investment. It is clear that mutation and limited local interactions are crucial in promoting (even to keep) cooperation in the spatial CPD. In fact, if each individual interacts with the whole population and there is no mutation, in the next step, each individual always will invest the lowest value from the initial generation. However, as occurs in the standard Prisoner's Dilemma on regular networks $[21,25]$, we argue for the spatial CPD that the spatial structure is capable of sustaining cooperation only in limited cost/benefit regimes. To study this issue, we consider the spatial CPD on a square lattice with periodic boundary conditions, with individuals interacting with their four nearest neighbors (von Neumann neighborhood) and synchronous updating.

\section{Asymptotics for different cost regimes}

Let us analyze the evolution of the social network defined in Section 2 in the simplest scenario. First, we consider an initial condition with a single individual that invests $x<y$ in a group of $y$-individuals (individuals who invest $y$ ) and assume no mutations. In that case, the update rule is deterministic and we can compute analytically the evolution of the system. More precisely: (i) If $C(y)-C(x)<(B(y)-B(x)) / 4$, the $x$-individual invades only its four nearest neighbors.

(ii) If $(B(y)-B(x)) / 4<C(y)-C(x)<(B(y)-B(x)) / 2$, the $x$-individual spreads over the lattice, reaching a structure with the same shape of the cross with sawtooth boundaries showed by [25].

(iii) If $C(y)-C(x)>(B(y)-B(x)) / 2$, the $x$-individual spreads until the extinction of the $y$-individuals.

Assuming that cost and benefit are differentiable functions, and using the fact that they are increasing and concave, it is easy to establish

$$
\frac{C^{\prime}(1)}{B^{\prime}(0)}<\frac{C(y)-C(x)}{B(y)-B(x)}<\frac{C^{\prime}(0)}{B^{\prime}(1)}
$$

for all $0<x<y<1$. Thus, the quotients $C^{\prime}(1) / B^{\prime}(0)$ and $C^{\prime}(0) / B^{\prime}(1)$ provide sufficient and necessary conditions, regardless of the values $x$ and $y$, to determine which absorbing state is reached. For all $0<x<y<1$, from a lattice of $y$-individuals, except one who that invests $x$, the condition $C^{\prime}(0)>B^{\prime}(1) / 4$ is necessary for the spread over all the lattice of the smallest investment and the condition $C^{\prime}(1)>B^{\prime}(0) / 4$ is sufficient. Additionally, $C^{\prime}(1)>B^{\prime}(0) / 2$ is sufficient for the extinction of the highest investment. In our study, we discriminate accordingly the different payoff regimes:

- Low cost, when $C^{\prime}(0)<B^{\prime}(1) / 4$.

- Low-medium cost, if $B^{\prime}(1) / 4<C^{\prime}(0)$ and $C^{\prime}(1)<$ $B^{\prime}(0) / 4$.

- High-medium cost, if $C^{\prime}(1)>B^{\prime}(0) / 4$.

- High cost, if $C^{\prime}(1)>B^{\prime}(0) / 2$. 
For simplicity, sometimes we will not distinguish between low-medium and high-medium costs and we refer to both as medium costs. Notice that if the benefit is a linear function, namely $B(x)=B x$, the only low-medium cost is the linear function $C x$, with $C=B / 4$. In that case, $B / 4$ and $B / 2$ are threshold values to determine the region of low, medium and high costs.

\subsection{Asymptotics from random initial conditions} without mutation

If a lower-investing individual spreads into a lattice of higher-investing individuals), two lower-investing individuals do the same. Furthermore, for high-medium costs, if there are different low investments into a lattice of higherinvesting individuals, at least the lowest spreads over the whole system and other low investments can locally do the same. If the initial investments are randomly chosen, the previous argument, and the fact that investments are initially well-mixed on the network, allow us to conjecture that high-medium cost is sufficient for the spreading of lower investments from random initial conditions. On the other hand, as we argued above, at high costs the smallest investment always dominates the network. In contrast, if the cost is low, the small investments do not spread on the system and limited local interactions of the lattice are capable of maintaining cooperation. If the cost is lowmedium, we do not provide arguments for determining the asymptotic behavior of the system from a random initial condition. For this reason, it is advisable to first take a look at the linear benefit case, where low-medium costs are reduced only to one threshold value.

The type of social network that we are studying typically reaches a steady state where the mean investment lightly fluctuates around a value, called the asymptotic mean investment $[17,19]$. Thus, the promotion or sustaining of cooperation can be contrasted by comparing the asymptotic mean investment with the initial one. Figure 1 shows the typical evolution in time of the mean investment as well as the investment configuration at the steady state for the different cost regimes. For this illustration, we considered the same random initial configuration, uniformly distributed on $[0,1]$ random variables placed on the $100 \times 100$ lattice. For low costs, the figure displays the previously reported clusterization of high investments and consequent promotion of cooperation. However, the figure also illustrates the spread of small investments for medium cost regimes as well as the domination of the lowest for high costs. That is, the square lattice is only capable of promoting (even sustaining) cooperation in low cost regimes.

To have an overview of the asymptotic behavior of the investments for different costs we considered different basis functions and varied the cost along its range. For each parametric set, we perform simulations for 100 initial random configurations until the steady state. Although the asymptotic mean investment depends on the initial condition, we noted that its slope, in terms of the cost, is strongly independent. For this reason, we use the average, over the initial random conditions, as a smooth in- 

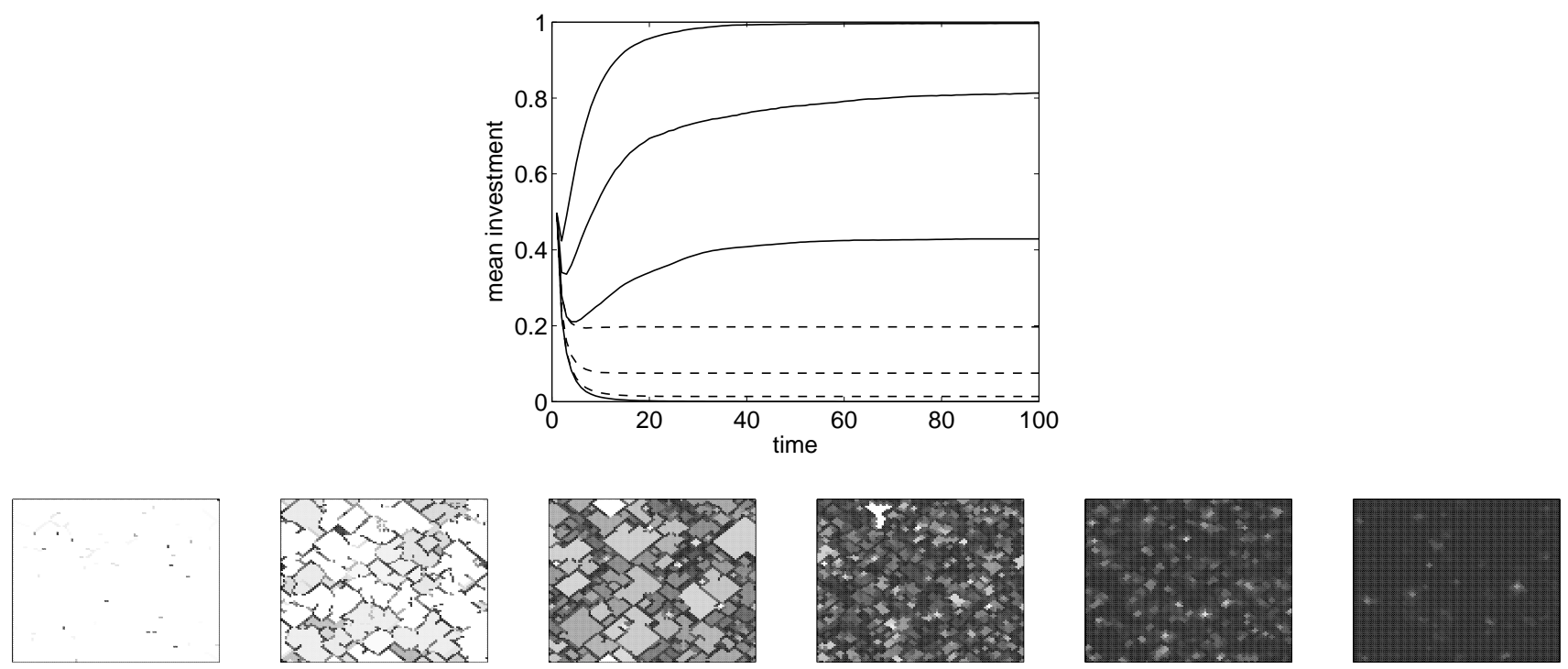

Fig. 1. Time series of mean investments and configuration of the lattices at the steady state for linear benefit and low (solid lines), medium (dashed lines) and high (bottom solid line) costs, from the same random initial investments. The figure illustrates the typical behavior close to the threshold costs and in the middle values. The top solid lines and the first three snapshots correspond, respectively, to low costs, $\left\{\delta, \frac{1}{8} B, \frac{1}{4} B-\delta\right\}$, with $\delta=B / 1000$. The dashed lines and the last three snapshots to medium costs $\left\{\frac{1}{4} B+\delta, \frac{3}{8} B, \frac{1}{2} B-\delta\right\}$. The bottom solid line corresponds to high cost $\frac{1}{2} B+\delta$ (its snapshot at the steady state is omitted, a complete black square). The gray scale used to represent the investments on the lattice is linear between black $=$ 0 and white $=1$.

dicator of the relation between costs and the asymptotic mean investment. The slope for four illustrative cases are displayed in Figure 2. We remark that for costs above the threshold cost $B^{\prime}(0) / 2=a b / 2$ the lowest investment dominates the network from any random initial conditions. Note that the transition between significant high asymptotic levels of cooperation to low levels occurs in low-medium costs, namely $B^{\prime}(1) / 4<C<B^{\prime}(0) / 4$. This transition is discontinuous if the benefit is the linear function $B x$. In that case, the phase transition occurs at the threshold cost $B / 4$. The bifurcation of the mean investment time series around this critical cost can be observed

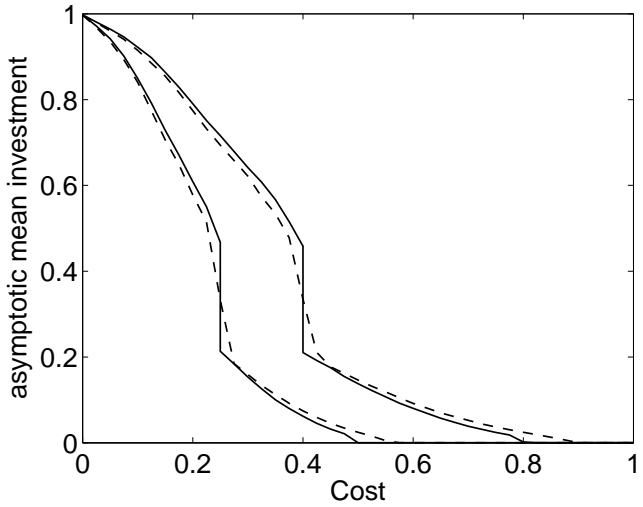

Fig. 2. Asymptotic mean investment as function of the cost. The left solid line corresponds to the linear benefit $B(x)=x$ and the right one to $B(x)=8 x / 5$. Left dashed line corresponds to the benefit function $B(x)=5(1-\exp (-2 x / 9))$ and the right one to $B(x)=8(1-\exp (-2 x / 9))$. 
in Fig. 1, by comparing the resulting time series for costs $B / 4-\delta$ and $B / 4-\delta$, with small $\delta$.

\subsection{Asymptotic from small initial investment with mutation}

The mutation mechanism introduced by [12] corresponds to neutral variations in the investment pool. Specifically, they consider normal errors, at the imitation moment, with variance equal to $10 \%$ of the expected investment.

Without mutation, the mean investment of the network remains into the range of the initial investments. The main result of [12] is that starting from arbitrary low investments, occasional mutations can increase the mean investment to significant levels, after a long runtime. Their simulations are based on a parametric set that we have characterized as low cost. Namely, $B(x)=8(1-\exp (-x))$, $C(x)=0.7 x$, and $x_{\max } \approx 2.4361$. Performing simulations with the same mutation rate per individual (1/100) and modeling errors in the same way, we confirm their main result for any low cost, when the benefit function is not linear. Moreover, we are now prepared to explain why mutation raises cooperation for this cost/benefit regime: When costs are low, our results show that negative mutations (i.e. mutations with lower investments than their expectations) do not spread on the system and that higherinvesting clusters invade lower-investment ones. To see this, notice that individuals in the boundaries of the higherinvesting cluster outperform the individuals in the boundaries of the lower-investing cluster. On the other hand, interactions with eventual positive mutations (mutations with investments higher than their expectations) can give origin to a small cluster of higher investments, again in the low cost regime. For a strictly concave benefit function, the lower the investment the higher the additional payoff that a positive mutant adds to their neighbors. Additionally, the more concave the benefit function is, the stronger such effect is. To sum up, in this unbeatable cost/benefit framework (e.g. low cost and very concave benefit function), a positive mutant can gradually invade a world of low investors until obtaining a high mean investment at the steady state. However, the less concave the benefit function is the lower the mean investment reached is. This pattern coincides with the results of [18], who analyze different regimes of physiological costs in a related adaptive dynamics with the CPD. Our results complement the analysis for lattice-structured population. In fact:

1. If the benefit is linear, the lattice-structured population is only capable of sustaining the mean investment around the initial one, even if the cost is low.

When significant costs are considered, other asymptotic results occur:

2. If the cost is low-medium, for any basis benefit function (linear or strictly concave), the lattice is only capable of sustaining the mean investment around the initial one. This occurs with and without mutation. A mutation (negative or positive) does not have any effect on the lattice.

3. In high-medium cost regimes, the mutation mechanism works against cooperation, by favoring negative mutations (i.e. mutations with lower investments than the 
expectations). Thus, if there are not mutations, the lattice can sustain low levels of cooperation, but cannot stop the fall of investments if any occasional negative mutation appears on the lattice.

4. For high costs, the investments nose dive, with or without mutation.

Summarizing, small mutations promote cooperation only in very particular scenarios of low costs of cooperation.

\section{Effects of resistance to imitation on the evolution of cooperation}

The above analysis was obtained when the strategy update rule is based on exact imitation. Now we consider weighted average as the way to update investments. Instead of focal individuals copying the best neighbor, each individuals updates her investment with a new one "between" her own investment and the investment of her successful neighbor. Into the spatial CPD, weighted average operates as follows: If an individual invests $x$ and her successful neighbor invests $y$, in the next step, the former will invest

$$
\alpha x+(1-\alpha) y
$$

plus a possible error, for some $\alpha \in[0,1]$.

In an economic and social scenario, the parameter $\alpha$ can be viewed as a factor of resistance to imitation or as a measure of wariness. When $\alpha=0$, there is no resistance, individuals copy the strategy of their neighbors with the highest payoff. However, when $0<\alpha<1$ the individ- uals gradually learn from their most successful partner. Whereas if $\alpha=1$ individuals refuse to imitate.

\subsection{Supporting cooperation in adverse cost regimes}

As in Section 3, we analyze first the evolution in time of the social network without errors in the update rule. Similar to the previously studied case, the lattice reaches a steady state where the mean investment lightly fluctuates around a constant. Figure 3 shows the typical evolution in time of the mean investment for different resistance levels in medium and high costs. It is noteworthy how the presence of resistance to imitation can increase the asymptotic mean investment to higher levels when investments are costly.

To have a better understanding of the effect of resistance to imitation, we considered different cost/benefit functions and varied the resistance along its range. For each parametric set, we performed simulations for 100 initial random configurations until the steady state was reached. We observe that only small variations on the asymptotic mean investments were observed. Thus, the average, over the initial random conditions, is a robust estimator of this asymptotic mean investment, regardless the initial condition. In general, in low cost regimes, resistance neither favors cooperation nor dulls it. However, for any medium or high cost, resistance to exact imitation strongly favors cooperation. Moreover, the higher the resistance is the larger the mean investment asymptotically reached. Figure 4 characterizes the behavior for the different cost regimes. We conclude that cautious learn- 


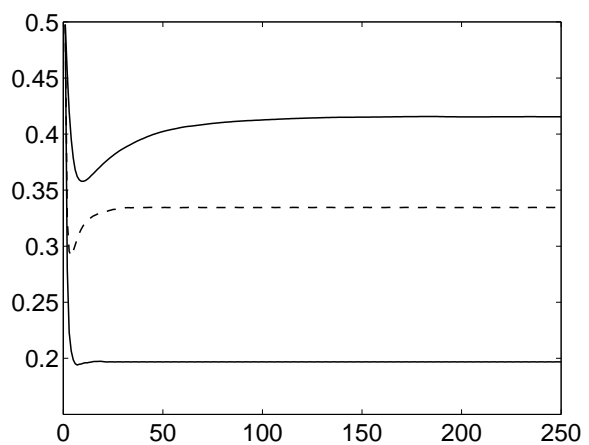

(a)

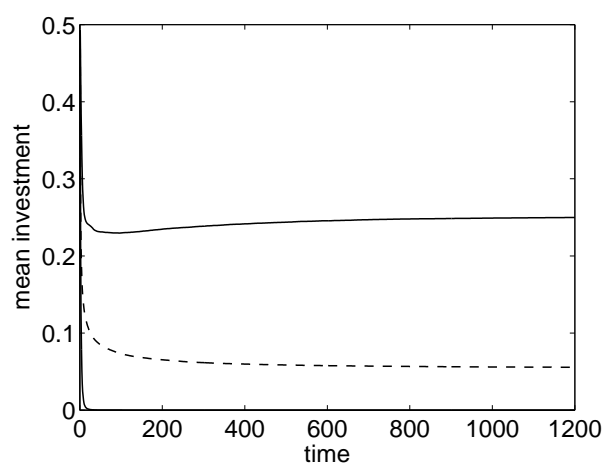

(b)

Fig. 3. Time series of mean investments for medium (a) and high (b) cost with different resistance degrees. Top solid lines correspond to $\alpha=0.8$, dashed lines to $\alpha=0.2$ and bottom solid line to $\alpha=0$. The benefit function is linear, and the costs considered are to the right of the thresholds $\frac{1}{4} B$ and $\frac{1}{2} B$.

ing is more efficient than hasty learning when the cost is medium or high and it is necessary to sustain cooperation (even in low levels) for high costs.

To understand how resistance works to favor cooperation in medium/high cost regimes, let us consider again the simplest initial condition with a single $x$-individual in a lattice occupied with $y$-individuals, with $x<y$. Notice that the $x$-investment spreads on the lattice (or dominates it) if individuals have no resistance to imitation. For this initial configuration, let the epicenter be the individual with initial investment $x$ and the front of the wave be the

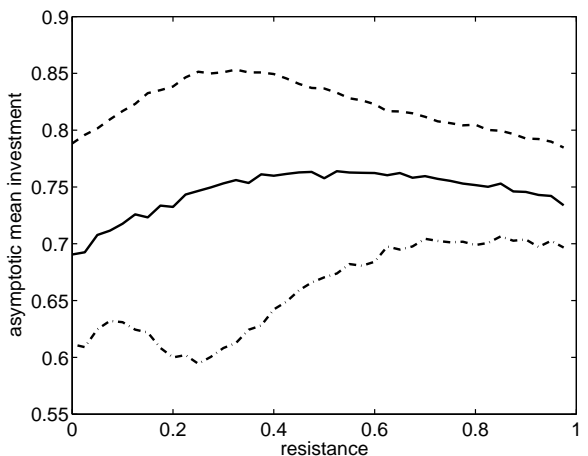

(a)

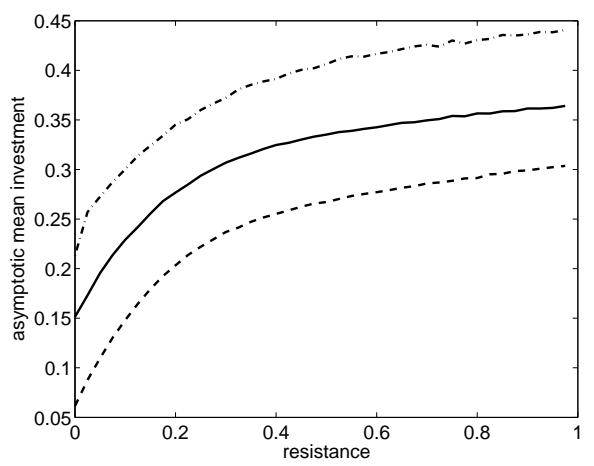

(b)

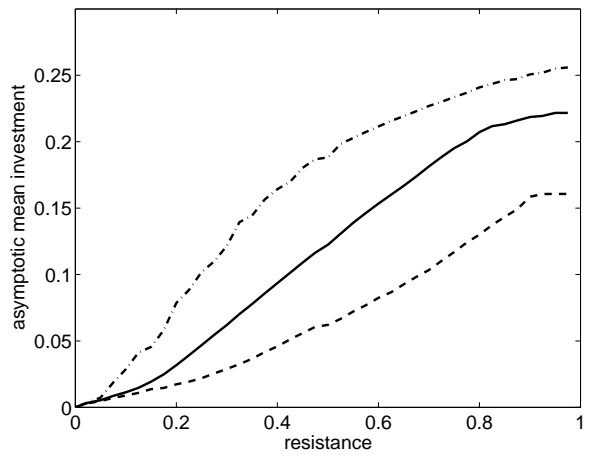

(c)

Fig. 4. Asymptotic mean investment as a function of $\alpha$ for the benefit function $B x$. Low costs in (a): top dashed line $\frac{1}{8} B$, solid line $\frac{1}{6} B$, and bottom dashed line $\frac{1}{5} B$. Medium costs in (b): top dashed line $\frac{1}{4} B+\delta$, solid line $\frac{3}{10} B$, and bottom dashed line $\frac{2}{5} B$. High costs in (c): top dashed line $\frac{1}{2} B+\delta$, solid line $\frac{3}{5} B$, and bottom dashed line $\frac{3}{4} B$. To consider costs close to the thresholds we make $\delta=1 / 1000$. 

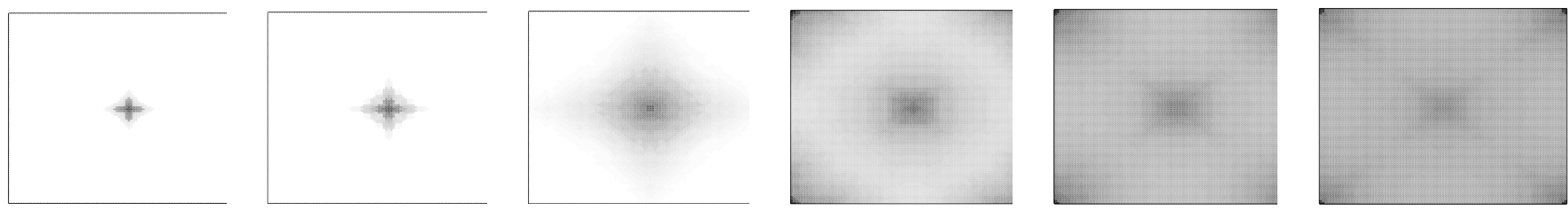

Fig. 5. Attenuation of a 0 -investor for a pool of 1-investors with low degree of resistance at time generations 10, 20, 100, 300, 1000 and 3000. $B(x)=4(1-\exp (-x / 2)), C=0.8$, and $\alpha=0.25$. The asymptotic mean investment in the stationary state is around 0.67. Note: for this parametric set, the 0-invertor dominates the system if there is no resistance.

$y$-individuals closer to the epicenter. It is easy to show that while individuals gradually learn from the individual with the least investment (expansion of the wave), the investment at the front of the wave of the generation $k$ changes to $y-(1-\alpha)^{k}(y-x)$ in the next step. Thus, if the lattice is big enough, there exists a resistance value such that these individuals at the front of wave learn from their neighbors with higher investments, in some generation, producing a reflection of the wave. An immediate consequence of this result is that the expansive wave generated by the $x$-agent is reflected by a barrier of $y$-individuals when those have enough resistance. The reflected wave is propagated towards the epicenter making an increment of the mean investment of the system. Thus, if the lattice is big enough and individuals have enough resistance to imitation, the system can asymptotically recover high investments (defection absorption); even if there is not enough resistance to imitation, the wave can be attenuated producing a diminishment in the mean investment (defection attenuation). Moreover, the absorption/attenuation effect, illustrated in Figures 5 and 6 , can locally operate for random initial configuration until producing the rebound in the mean investment evolution showed in Figure 3(a).

\subsection{The origin of cooperation: Learning errors and resistance to imitation}

The most amazing results occur when there are occasional errors in the gradual learning rule. To study this phenomenon, we performed simulations by covering low, medium and high costs and linear and strictly concave benefit functions. As in Section 3.2, we considered low initial investments, uniformly distributed between zero and $1 / 10$, and error rate of $1 \%$, Gaussian distributed with variance equal to $10 \%$ of the expected investment. Our study is conclusive as to the effect of the resistance to learning:

1. In medium costs (low-medium and high-medium) as well as in low costs with linear benefits, where the model with learning errors and without resistance is only capable of sustaining cooperation in the initial investment range, gradual learning leads to significant higher asymptotic mean investment.

2. In high costs, occasional errors work against cooperation and even the resistance to imitation is unable to maintain cooperation.

Figure 7 shows the typical effect of the resistance to imitation in adverse conditions in which simple mutations in 

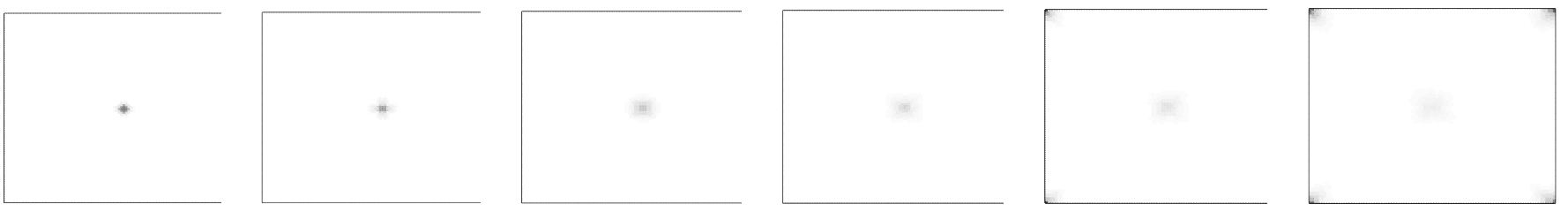

Fig. 6. Absorption of a 0-investor for a pool of 1-investors with high enough degree of resistance at time generations 10, 20, 100, 300, 1000 and 3000. $B(x)=4(1-\exp (-x / 2)), C=0.8, \alpha=0.65$. The asymptotic mean investment in the stationary state is almost 1 .

the structured population are not sufficient to trigger high levels of cooperation.

In these scenarios of low and medium costs, with linear or strictly concave benefits, there always exists a value for the resistance parameter to raise cooperation to higher levels. Moreover, we observed through simulations, that any resistance level $(0<\alpha<1))$ is enough to promote cooperation when costs are low. Medium levels of resistance may be required when costs are medium; the closer the cost is to the high cost threshold, the higher the level of resistance required.

\section{Conclusions}

In this paper we have studied the effect on the evolution of cooperation of introducing resistance to imitation in the strategy update rule of the spatial CPD. We have reviewed this evolutionary game in detail when the strategy update rule is based on unconditional imitation. First, we have studied the model without errors in the imitating process. For this case, we have found a simple classification of the payoff regimes (low, low-medium, high-medium, and high costs) which allows us to determine the asymptotic mean behavior of a population structured on a square lat-

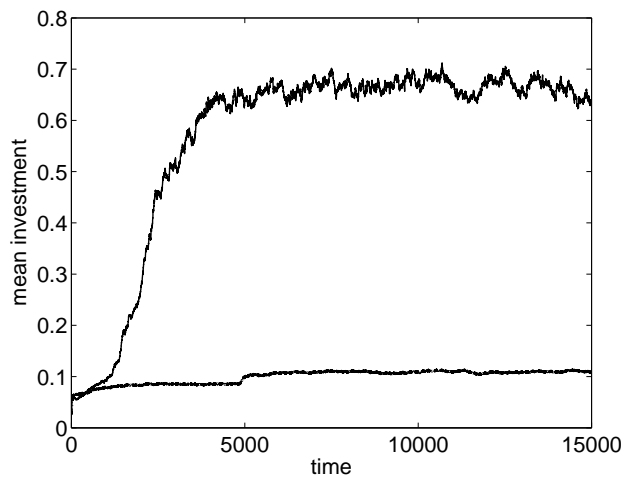

(a)

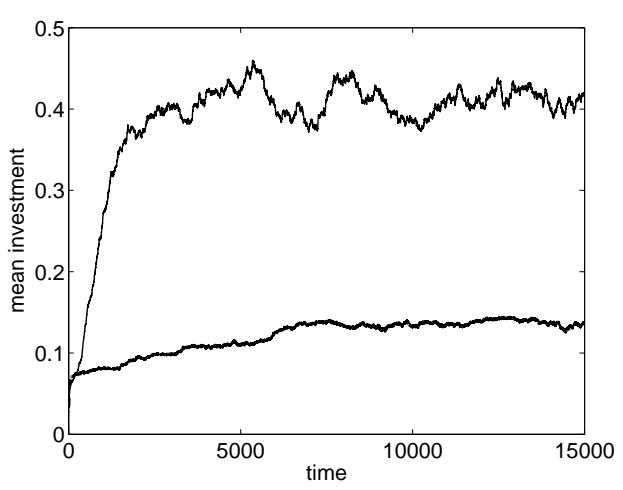

(b)

Fig. 7. Time series of mean investments with resistance (top lines) and without resistance (bottom lines): (a) linear benefit $(B=1)$, low cost $(C=0.2)$ and low resistance $(\alpha=0.1)$. (b) strictly concave benefit $(B(x)=4[1-\exp (-x / 2)])$, medium cost $(C=0.4)$ and medium resistance $(\alpha=0.5)$. Gaussian mutation, with variance equal to $10 \%$ of the expected investment and mutation rate $1 \%$. Initial investments are uniformly distributed on $\left[0, \frac{1}{10}\right]$. 
tice. From random initial conditions, the spatial structure promotes high investments only in low cost regimes. We observed fast transitions to low mean investments in lowmedium costs. It is interesting to notice that these transitions are discontinuous when the payoffs are linear functions. In regimes of high-medium cost the spatial structure is unable to keep the initial mean cooperation investment. The lowest investment in the initial state dominates the system for any high-cost regime. When occasional errors in the imitating process are considered, cooperation emerges from a non-cooperative initial state only under a particular regime of low cost and strictly concave benefit. To observe this emergence, previously reported in the literature of the spatial CPD [12,19], negligible costs of cooperation [18] are required. For the rest of the regimes, the essential problem of cooperation remains: Occasional errors have no effect on the evolution of cooperation when costs are low and the benefit function is linear or when costs are low-medium. Moreover, they work against cooperation in high-medium and high costs.

Weighted average can be viewed as a step-by-step learning mechanism in the strategy update process that can prevent individuals from their costly acts. Amazingly, it is this aspect that offers important insight into the problem of origin and sustainment of cooperation. Our analysis explains how cooperation evolves and is maintained when gradual learning works in the spatial CPD. We have proved that the resistance to exact imitation has a significant effect on medium (low-medium and high-medium) cost and high cost regimes: The less that is learnt in a single step, the greater the degree of global cooperation finally attained. When learning errors can occur on an arbitrarily non-cooperative population, we have showed that a higher-investment mutant spreads all over the system raising cooperation to significant levels in regimes of low costs (with linear or non-linear benefits) and medium costs. The larger the cost, the greater the resistance to imitation needed in a single step. However, in more adverse conditions of high costs, occasional errors work against cooperation and even the resistance is unable to maintain cooperation.

We call attention to the fact that gradual learning can be introduced in a straightforward way into the strategy update rule of any continuous evolutionary game, such as the continuous Snowdrift game [26] and the spatial ultimatum game [27]. Gradual learning can also operate jointly with different selection mechanisms to identify from whom to learn. To compare results with previous literature on spatial the CPD, we have considered learning from the successful neighbor, but the weighted average operator can be easily fitted to random selection criterions [7]. We have only considered weighted average, because it is the natural rule when strategies are represented by real numbers, such as cooperative investments. But different schemas of gradual learning can be considered when strategies are not scalar. Our formulation is quite simple and can be applied in a wide range of scenarios. We hope that this work will contribute to highlighting the relevant role of this evolutionary principle for the study of cooperation. 
In conclusion, resistance to imitation provides a natural solution to the evolutionary riddle of the origin and maintenance of cooperation in a world governed by selective forces. Our results suggest that populations which learn slowly from successful partners are, in the long term, more efficient in the sense that they allow for higher cooperative investments. Thus, our model provides an evolutionary version of the tortoise and the hare fable when the goal is cooperation.

We gratefully thank Víctor Eguíluz of IFISC for multiple inspiring discussions related to this paper. This work was supported by MEC (Spain) under grants ECO2008-05080, SEJ200767135 and FISICOS, CSIC (Spain) under grant PIE200750I016, and EU under grant GABA.

\section{References}

1. R. Trivers, Q. Rev. Biol. 46, 35-57 (1971).

2. R. Axelrod, W. D. Hamilton, Science 211, 1390-1396 (1981).

3. J. Maynard, E. Szathmáry, The Major Transitions in Evolution (Oxford: W. H. Freeman \& Co, 1995).

4. L. A.Dugatkin, Cooperation among animals (Oxford University Press, 1997).

5. M. A. Nowak, Science 314, 1560-1563 (2006).

6. E. Pennisi, Science 309, 93 (2005).

7. G. Szabó, G. Fáth, Phys. Rep. 446, 97-216 (2007).

8. G. Mar, P. St Denis, Int. J. Bifur. Chaos 4, 943-958 (1994).

9. M. Frean, J. Theor. Biol. 182, 549-559 (1996).

10. M. Doebeli, N. Knowlton, Proc. Natl Acad. Sci. USA 95, 8676-8680 (1998).
11. G. Roberts, T. M. Sherratt, Nature 394, 175-179 (1998).

12. T. Killingback, M. Doebeli, N. Knowlton, Proc. R. Soc. Lond. B 266, 1723-1728 (1999).

13. L. M. Wahl, M. A. Nowak, J. Theor. Biol. 200, 307-322 (1999).

14. L. M. Wahl, M. A. Nowak, J. Theor. Biol. 200, 323-338 (1999).

15. T. N. Sherratt, G. Roberts, G., J. Theor. Biol. 200, 419426 (1999).

16. J. C. Koella, J. C., Proc. R. Soc. Lond. B 267, 1975-1985 (2000).

17. T. Killingback, M. Doebeli, Am. Nat. 160, 421-438 (2002).

18. J. F. Le Gaillard, R. Ferriere, U. Dieckmann, Evolution 57, 1-17 (2003).

19. M. Ifti, T. Killingback, M. Doebeli, J. Theor. Biol. 231, 97-106 (2004).

20. T. N. Sherratt, G. Roberts, J. Theor. Biol. 215, 47-56 (2002).

21. M. Doebeli, C. Hauert, Ecol. Lett. 8, 748-766 (2005).

22. M. A. Nowak, R. M. May, Nature 415, 424-426 (1992).

23. G. S. Wilkinson, Nature 308, 181-184 (1984).

24. B. Hart, L. Hart, Anim. Behav. 44, 10731083 (1992).

25. R. Jiménez, H. Lugo, J. Cuesta, A. Sánchez, J. Theor. Biol. J. 250, 475-483 (2008).

26. M. Doebeli, C. Hauert, T. Killingback, Science 303, 859862 (2004).

27. K. M. Page, M. A. Nowak, K. Sigmund, Proc. R. Soc. Lond. B 267, 2177-2182 (2000). 